\title{
LIMIAR DE SENSIBILIDADE CUTÂNEA DOS PÉS EM PACIENTES DIABÉTICOS ATRAVÉS DO PRESSURE SPECIFIED SENSORY DEVICE: UMA AVALLAÇÃO DA NEUROPATIA
}

\author{
Viviane Fernandes de Carvalho*1, Marcus Castro Ferreira ${ }^{2}$, Suzy Ane Tavares Vieira ${ }^{3}$, Thiago Ueda ${ }^{4}$ \\ Trabalho realizado pela disciplina de Cirurgia Plástica da Faculdade de Medicina da Universidade de São Paulo
}

\author{
*Correspondência: \\ Faculdade de Medicina da \\ USP \\ Laboratório de Microcirurgia \\ e Cirurgia Plástica \\ Avenida Doutor Arnaldo, \\ $455-1^{\circ}$ andar - Sala 1360 \\ CEP $01246-903$ \\ São Paulo - SP \\ Tel: (11) 3062-2116/ \\ Fax: (11) 3062-0415 \\ vivianefcarvalho@usp.br
}

\begin{abstract}
RESUMO
OBjetrvo. A neuropatia diabética leva à diminuição ou perda da sensibilidade protetora do pé, tornando o diabético mais vulnerável ao trauma mecânico, consequentemente, levando-o à formação de feridas e eventualmente, perda segmentar nos membros inferiores. A profilaxia das complicações neuropáticas deve ser iniciada pela identificação do grau de neuropatia e, portanto, do comprometimento neurológico. O Pressure Specified Sensory Device ${ }^{\text {Th }}$ foi desenvolvido para quantificar o limiar de pressão aplicada sobre a pele, necessário para que o paciente sinta o estímulo de um ponto estático, um ponto em movimento, dois pontos estáticos e dois pontos em movimento. É um meio direto para se avaliar os sistemas de fibras de adaptaçãa lenta e rápida e seus respectivos receptores periféricos.

Métodos. Trinta e três pacientes diabéticos do tipo II, sem história prévia de feridas e/ou amputações nos pés foram avaliados neste estudo de corte transversal. A sensibilidade nos territórios cutâneos dos nervos plantar medial, calcâneo e o ramo profundo do nervo fibular foi avaliada usando os testes de um ponto estático (IPE), um ponto dinâmico (IPD), dois pontos estáticos (2PE) e dois dinâmicos (2PD).

REsULtADOS. Nos três territórios nervosos examinados encontramos valores alterados para as modalidades estática e dinâmica em relação ao padrão de normalidade. As diferenças foram estatisticamente significantes com $p<0,05$.

Conclusão. A quantificação numérica do limiar de pressão cutânea nos permite tanto a avaliação do conjunto fibra/receptor, quanto à determinação do déficit funcional da fibra nervosa imposto pela neuropatia diabética.

UnTtermos: Neuropatias diabéticas. Sensibilidade cutânea. Métodos de avaliação.
\end{abstract}

\section{INTRODUÇÃo}

A neuropatia diabética diminui ou causa perda da sensibilidade protetora dos pés, tornando este segmento mais vulnerável ao trauma, consequentemente, poderá levar à formação de feridas ou até amputação parcial ou total dos membros inferiores (MMII)' .

Segundo Dyck et al. ${ }^{2}$ a perda da sensibilidade protetora causada pela neuropatia diabética, em contraposição a outras doenças que também lesam nervos periféricos, é um processo irreversível. Foram descritas técnicas cirúrgicas e procedimentos clínicos para se evitar a progressão dos danos provocados pela neuropatia diabética, entretanto não há consenso sobre qual seria o tratamento idea $\left.\right|^{3,4,5}$. A maioria dos especialistas indica medidas de prevenção, como a melhor forma de se conter as consequências impostas pela neuropatia diabética ${ }^{6,7,8,9,10}$.

A profilaxia das complicações neuropáticas deve ser iniciada pela identificação do grau de neuropatia e, portanto, do déficit neurológico. Isto é possível, atualmente, por meio de avanços na avaliação de sensibilidade cutânea nos MMII. Para Dellon et al. " , a metodologia dos testes de sensibilidade evoluiu da observação qualitativa para a quantificação de variáveis numéricas.

Os principais testes disponíveis para examinar a perda funcional de sensibilidade cutânea estão relacionados à sensação táctil, representada pelas respostas à pressão e à vibração. Esta qualidade de sensação é mediada por axônios mielinizados, conhecidos como fibras de adaptação lenta e rápida, conforme seu receptor periférico ${ }^{12}$.

Em 1898, von Frey introduziu um método clínico para avaliação da sensibilidade da pele ao tato. Pressionando a pele com o "filamento de von Frey", registrou a sensibilidade à pressão observando o limiar mínimo obtido a cada filamento utilizado no teste ${ }^{13}$.

Em 1962, Weinstein aperfeiçoou o método de von Frey, adaptando os monofilamentos de nylon em hastes plásticas. Este método conhecido atualmente como monofilamento de Semmes-Weinstein ${ }^{\circledR}$ é tido como padrão-ouro para medidas de sensibilidade das fibras de adaptação lenta, devido à simplicidade, rapidez e baixo custo do teste ${ }^{14}$.

Para avaliar a função das fibras de adaptação rápida e seus receptores periféricos são usadas manobras com instrumentos produtores

1. Doutora em Ciências pela FMUSP. Área de concentração

2. Professor Titular da Disciplina de Cirurgia Plástica FMUSP. São Paulo,SP, Brasil

3. Mestre em Ciências pela FMUSP. Área de concentração Cirurgia Plástica. São Paulo, SP, Brasil

4. Aluno de graduação do curso médico da Faculdade de Medicina da USP; Bolsista de iniciação científica FAPESP. São Paulo, SP, Brasil 
de vibração, por exemplo diapasões com frequências de 30 a 256 ciclos por segundo ${ }^{15}$. Associando o exame de sensibilidade vibratória realizado com o diapasão de $128 \mathrm{~Hz}$ e a avaliação de sensibilidade pressórica, dada pelo monofilamento de Semmes-Weinstein ${ }^{\circledR}$, estudos mostram sensibilidade de $100 \%$ e especificidade de $77 \%$ para evolução da neuropatia diabética ${ }^{16}$.

Dellon ${ }^{17}$, em 1992, desenvolveu o Pressure-Specified Sensory Device $^{\mathrm{TM}}$ - PSSD (Sensory Management Services LLC, Baltimore, Maryland). Este dispositivo é capaz de quantificar o limiar de pressão aplicada sobre a pele necessário para que o paciente sinta o estímulo de um ponto estático, um ponto em movimento, dois pontos estático e dois pontos em movimento. É um meio mais preciso para avaliar os sistemas de fibras de adaptação lenta e rápida e seus respectivos receptores periféricos.

A disciplina de Cirurgia Plástica - FMUSP vem utilizando o PSSD ${ }^{\text {TM }}$ desde 2000 para determinação da sensibilidade cutânea em diversas situações. Ferreira et al. ${ }^{18}$ avaliou a sensibilidade cutânea no pré e pós-operatório de mastoplastia redutora. Estudo preliminar sobre a sensibilidade cutânea nos pés de pacientes diabéticos foi apresentado por Ferreira et al. ${ }^{19}$ em 2004. Devido ao reduzido número de trabalhos quantitativos sobre o limiar de pressão cutânea em nosso meio, a aplicação do PSSD ${ }^{\mathrm{TM}}$ contribuiu para determinação destes valores em regiões anatômicas como abdome ${ }^{20}$ e face ${ }^{21}$, locais frequentemente submetidos a procedimentos cirúrgicos e a traumas externos.

O presente estudo tem por objetivo quantificar o limiar de pressão cutânea dos pés de pacientes diabéticos em nosso meio através do Pressure-Specified Sensory Device ${ }^{\mathrm{TM}}$ - PSSD.

\section{Métodos}

Estudo de corte transversal, tipo observacional, seguido de análise estatística descritiva ${ }^{22}$. Realizado no Instituto Central do Hospital das Clínicas da Faculdade de Medicina da USP em pacientes regularmente matriculados no Ambulatório de Feridas Complexas da Disciplina de Cirurgia Plástica da referida instituiçãa. Aprovado pela Comissão de Ética para Análise de Projetos de Pesquisa (CAPPesq) em 10/05/2006, sob o número protocolar 356/06.

Os pacientes considerados aptos a participar do estudo foram aqueles que atendiam aos critérios de inclusão:

- Idade mínima de 18 anos;

- Portador de Diabetes melitus tipo II, segundo critérios adotados pela Sociedade Brasileira de Diabetes, ${ }^{23}$

- Não ter apresentado, até o momento, feridas nos MMII;

- Não ter sido submetido à amputação nos MMII;

- Não apresentar doença vascular periférica;

- Não apresentar lesão neurológica que comprometesse função nervosa periférica;

- Ser capaz de responder, de maneira adequada, sobre seu estado de saúde.

Após explicação detalhada sobre o estudo e obtenção do termo de consentimento livre e informado, o paciente era interrogado sobre sua história clínica, como: duração do Diabetes melitus, tratamentos anteriores e atuais, doenças associadas e hábitos de vida.

A avaliação da sensibilidade nos MMII obedeceu à sequência proposta por Tassler et al. ${ }^{24}$. Os sítios anatômicos avaliados são aqueles
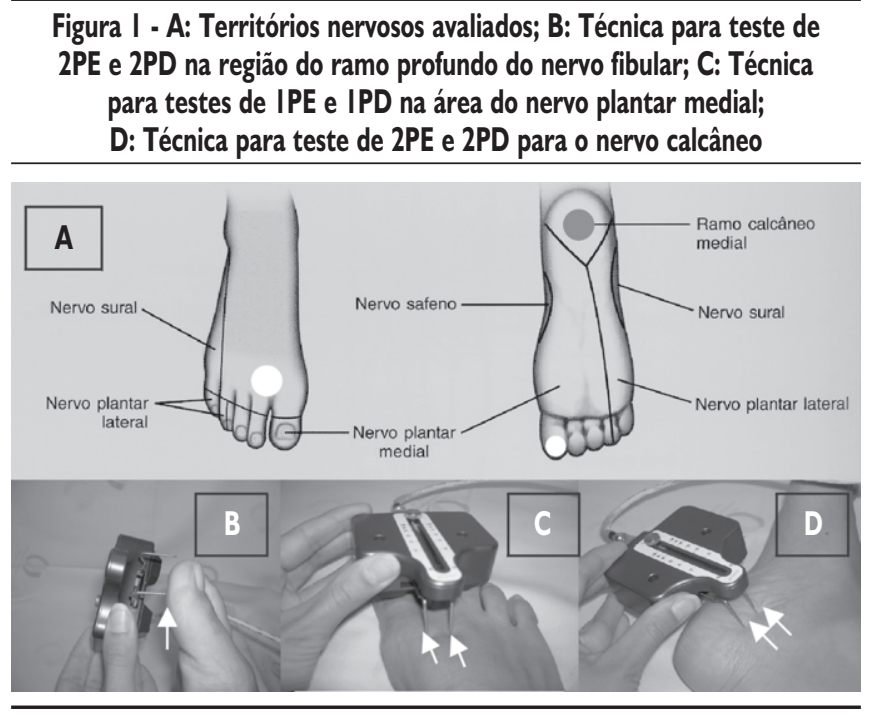

inervados pelos ramos do nervo tibial posterior: nervo plantar medial (polpa do hálux) e nervo calcâneo, além da região do dorso do pé, inervado pelo ramo profundo do nervo fibular (Figura IA).

Testes prévios foram realizados na mão do lado dominante do corpo para que o paciente entendesse a dinâmica do exame. Estavam orientados a manter os olhos fechados, a fim de evitar confusão pela sensação visual.

O exame com o PSSD ${ }^{T M}$ consiste no toque de dois prolongamentos de metais ligados a um transdutor de força. Este transdutor é acoplado a um software, o qual nos fornece a pressão exercida pelo toque à pele do paciente em gramas $/ \mathrm{mm}^{2}$.

Para o teste de um ponto estático (IPE) e um ponto dinâmico (IPD), apenas um dos prolongamentos entra em contato com a área a ser testada (Figura IB). Quando do teste de dois pontos estáticos (2PE) e dois dinâmicos (2PD), ambos os prolongamentos metálicos são tocados na pele do paciente (Figura IC e ID). Nos testes dinâmicos as pontas dos prolongamentos são arrastadas no sentido longitudinal da área avaliada. A distância entre os prolongamentos é estabelecida a partir da discriminação de dois pontos realizada por meio do DiskCriminator $^{\circledR}$.

Avaliados 33 pacientes, os quais atendiam aos critérios de inclusão. Devido à aplicação do PSSD ${ }^{\text {TM }}$ ser feita em três regiões anatômicas bilateralmente, 198 territórios nervosos foram examinados.

A idade média dos pacientes investigados foi de $61,05 \pm 11,2$ anos. Houve prevalência do sexo feminino $(51,5 \%)$ e indivíduos brancos (72\%).

Durante a anamnese, os pacientes foram interrogados a respeito do tempo de conhecimento da doença e comorbidades associadas. 0 tempo médio de conhecimento do Diabetes melitus foi de I3,5 \pm 6,08 anos. Em relação às comorbidades, a hipertensão foi a mais frequente, referida por $81,81 \%$ dos pacientes.

Os dados oriundos da anamnese e do teste de sensibilidade cutânea pelo PSSD ${ }^{\mathrm{TM}}$ foram submetidos à análise matemática descritiva pelo software Minitab - versão |4. I . A significância estatística foi considerada para níveis de $p<0,05$. 


\section{Resultados}

A avaliação da discriminação entre os dois pontos, estático (D2PE) e dinâmico (D2PD), para este grupo de pacientes obteve os seguintes resultados: ramo profundo do nervo fibular D2PE I2,20 $\pm 2,75 \mathrm{~mm}$ e D2PD I2,20 \pm 2,80 mm, nervo plantar medial D2PE I I,80 $\pm 3,25$ mm e D2PD I I,75 $\pm 3,30$ mm, nervo calcâneo D2PE I2,45 $\pm 3,70$ $\mathrm{mm}$ e D2PD I 2,30 $\pm 3,30 \mathrm{~mm}$.

Os dados apresentados no Gráfico I são referentes ao limiar de sensibilidade cutânea da comissura do primeiro e segundo dedos no dorso do pé, inervada pelo ramo profundo do nervo fibular. O resultado da média (md) \pm desvio padrão (dp), limiar mínimo (mín.), limiar máximo (máx.), assim como os valores do intervalo de confiança (95\%) encontrados estão expressos em gramas por milímetro quadrado $\left(\mathrm{g} / \mathrm{mm}^{2}\right)$.

A média \pm desvio padrão para um ponto estático (IPE) foi de 8,75 \pm 6,20 e IC [5,8; I2,4], um ponto dinâmico (IPD) 6,90 $\pm 5,35$ e IC $[4,9 ; 9,3]$, dois pontos estáticos (2PE) 7,55 $\pm 5,60$ e IC $[5,1 ; 10,2]$ e dois pontos dinâmico (2PD) $5,85 \pm 3,10$ [4,5;7, I], com $p<0,05$.

Os valores referentes à região inervada pelo nervo plantar medial expressas sob a forma média \pm desvio padrão foram: para um ponto estático (IPE) foi de $9,40 \pm 7,15$ e IC [6,5; I2,0], um ponto dinâmico (IPD) 8,85 \pm 6,05 e IC [6,5; II,4], dois pontos estáticos (2PE) I0,95 $\pm 6,65$ e IC $[6,5 ; 16,9]$ e dois pontos dinâmicos (2PD) 7,35 $\pm 3,05$ e IC [5,7;8,7], com $p<0,05$, visualizados no Gráfico 2 A.

Para a região sob inervação do nervo calcâneo, os valores obtidos para o limiar cutâneo de pressão de um ponto estático (IPE) foram de $9,50 \pm 5,90$ e IC $[7,3 ;$ II,0], um ponto dinâmico (IPD) 8,05 \pm 5,40 e IC [5,8;10,4], dois pontos estáticos (2PE) 10,40 $\pm 4,55$ e IC $[6,5 ; \mid 4,5]$ e dois pontos dinâmico (2PD) 7,75 $\pm 4,30$ e IC [4,6; |0,5], com $p<0,05$, conforme Gráfico 2 B.

\section{Discussão}

A detecção da perda de sensibilidade protetora é importante para identificar pacientes diabéticos que apresentam neuropatia periférica, e assim planejar reduzir o risco de desenvolvimento de feridas e eventuais amputações nos membros inferiores 25, 26, 27

O Consenso de San Antonio, realizado em 1988 pela American Diabetes Association, recomenda que o grau de comprometimento da função de fibras nervosas sensitivas seja avaliado quantitativamente, ao menos, uma vez por ano ${ }^{28}$.

Segundo Singh et al. ${ }^{10}$ todos os pacientes diabético devem ser avaliados quanto ao risco de ulceração nos pés. A avaliação inclui rigorosa anamnese, exame físico dos MMII e, especialmente, teste para verificar sensibilidade protetora por meio do monofilamento de Semmes-Weinstein ${ }^{\circledR}$.

A avaliação da sensibilidade é, usualmente, feita pelos monofilamentos de Semmes-Weinstein ${ }^{\circledR}$, método de fácil aplicação, não-invasivo e de baixo custo ${ }^{29}$, mas, estudos têm mostrado que não está claro qual espessura de filamento deve ser usada $30,31,32$, assim como não é exato o número de sítios anatômicos a serem pesquisados ${ }^{33}$ para determinar o risco de ulceração nos pés.

As críticas ao monofilamento de Semmes-Weinstein ${ }^{\circledR}$ são justificadas, uma vez que o estímulo nervoso produzido pelo toque não é

\section{Gráfico I: Limiar de sensibilidade cutânea para 0 ramo profundo do nervo fibular}

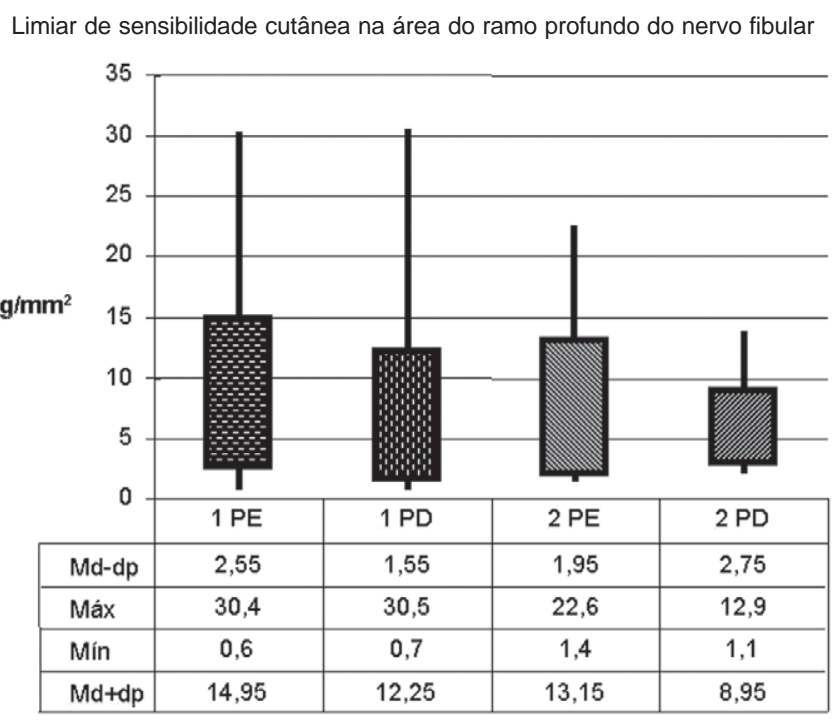

interpretado de maneira uniforme, pois áreas da pele cada vez maiores são tocadas devido aos diferentes e crescentes diâmetros dos filamentos. $O$ conjunto de 20 filamentos vem marcado por números em intervalos não regulares $(1,65$ a 6,65). Estes números são entendidos, erroneamente, como o valor da força em gramas. Representam, na realidade, o logaritmo na base 10 da força necessária para deformá-lo. O monofilamento deve ser aplicado perpendicularmente à superfície do corpo e a pressão é aumentada até que o filamento forme um arco. Este teste não quantifica a pressão necessária para percepção do tato, embora avalie, de forma grosseira, a função do conjunto fibra/receptor de adaptação lenta. Assim, o monofilamento é capaz de fornecer somente uma estimativa do limiar de pressão cutânea ${ }^{34}$.

Estudo realizado em $1978^{35}$ estabeleceu a correlação entre o número marcado no monofilamento de Semmes-Weinsten ${ }^{\circledR}$ e a pressão exercida para deformá-lo quando em contato com a pele, conforme Tabela I.

O Pressure-Specified Sensory Device ${ }^{\text {TM }}$ - PSSD quantifica o limiar de pressão cutânea de forma direta. Todavia, assim como ocorre com o monofilamento de Semmes-Weinstein ${ }^{\circledR}$, deve ser considerado subjetivo, uma vez que depende da informação do paciente se o toque na superfície da pele foi ou não sentido. Outro ponto a ser lembrado em relação ao PSSD $^{\text {TM }}$ é o custo para aquisição e treinamento necessário para utilização do equipamento.

O PSSD ${ }^{\text {TM }}$ apresenta resultados mais fidedignos que outros métodos, pois os prolongamentos metálicos que encostam na pele têm diâmetro constante, desta maneira a força exercida é compreendida como um estímulo regular. $O$ transdutor acoplado ao software exprime o valor da pressão (gramas $/ \mathrm{mm}^{2}$ ) necessária, em cada território nervoso, para a percepção do toque $e^{19,23}$.

O dispositivo PSSD ${ }^{\text {TM }}$ avalia a sensibilidade cutânea baseado na fisiologia das fibras nervosas e seus receptores de superfície. Fibras que geram impulsos instantaneamente à pele ser estimulada e continuam a 
Gráfico 2 - Registro dos limiares de sensibilidade cutânea para os nervos plantar medial (A) e calcâneo (B)

Limiar de sensibilidade cutânea na área de inervação do nervo plantar medial

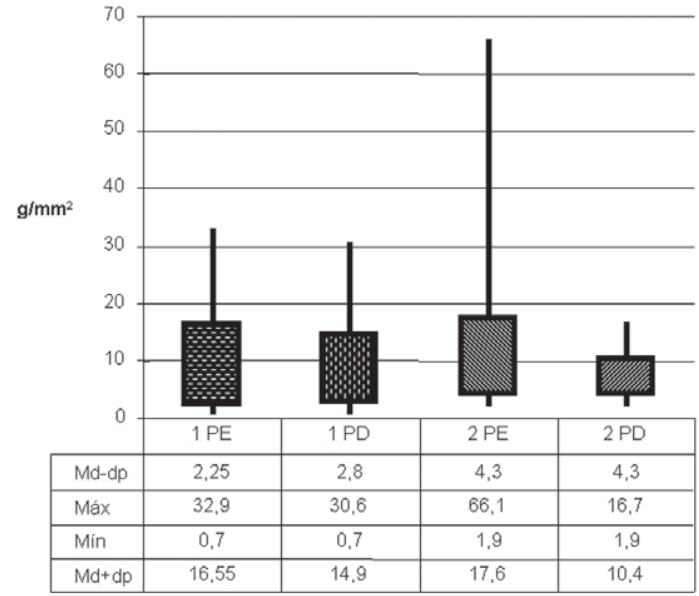

Gráfico 2A
Limiar de sensibilidade cutânea para o território inervado pelo nervo calcâneo

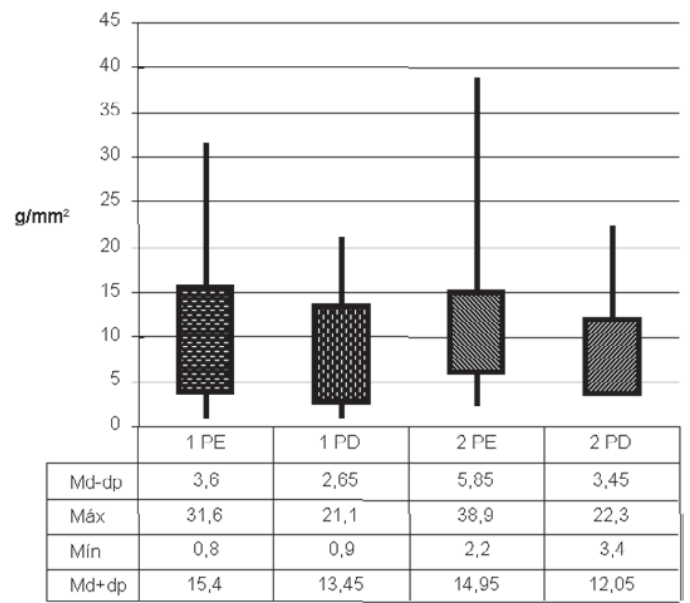

Gráíco $2 B$
Tabela I - Correlação entre o monofilamento de Semmes-Weinstein ${ }^{\circledR}$ e valores de pressão quando tocados na pele

\begin{tabular}{lcc}
\hline $\mathbf{N}^{0}$ filamento & $\mathbf{N}^{0}$ marcado no filamento & Pressão $\mathbf{( g / m m 2})$ \\
\hline 1 & 1,65 & 1,45 \\
4 & 2,83 & 4,86 \\
5 & 3,22 & $\mid 1,1$ \\
9 & 4,17 & 31,5 \\
10 & 4,21 & 33,1 \\
14 & 5,07 & 65,6 \\
\hline
\end{tabular}

gerá-los pelo tempo em que o estímulo é mantido em contato com a pele são classificadas como fibras de adaptação lenta. O estímulo é percebido como um toque constante e estático. Caso aumente a intensidade da força aplicada, haverá aumento da geração de impulsos e isto será percebido como um aumento de pressão. A integridade e a densidade destas fibras são medidas pelo limiar cutâneo de pressão, pela discriminação de um e dois pontos estáticos. Dois subtipos destas fibras podem ser distintos eletrofisiologicamente, tipo l: apresenta o comportamento descrito acima e tem como receptor as células de Merkel; tipo II: tem uma descarga espontânea regular e responde ao estiramento lateral da pele. Fibras do tipo II são denominadas de fibras de Ruffini. Na pele glabra o exato receptor ainda não foi identificado.

As fibras de adaptação rápida são aquelas que geram somente um ou dois impulsos ao serem incitadas e param de gerá-los tão logo o estímulo é interrompido. A capacidade destas fibras em gerar impulsos está ligada à percepção de novos estímulos, não à intensidade dos mesmos. A integridade e a densidade destas fibras são medidas pela distinção de um e dois pontos em movimento ou por vibração. Os corpúsculos de Vater-Pacini e Meissner são receptores associados às fibras de adaptação rápida em pele glabra e o folículo piloso, o receptor correspondente na pele não glabra.
Para estimar a densidade de fibras nervosas de forma não-invasiva, foi descrito por Weber ${ }^{36}$, em 1835, e Moberg ${ }^{37}$ a discriminação entre dois pontos, correspondente à distância mínima medida em milímetros reconhecidos pelo paciente, que sente dois pontos separadamente quando um clipe de papel é pressionado levemente sobre a superfície da pele do paciente. Posteriormente, Mackinnon e Dellon ${ }^{38}$ criaram o Disk-Criminator ${ }^{\circledR}$ para melhor padronizar esta estimativa.

Em relação aos nossos resultados, a região inervada pelo ramo profundo do nervo fibular, a comissura entre o primeiro e segundo dedos no dorso do pé, revelou limiares maiores para IPE e IPD, se comparados aos limiares de pressão estabelecidos para indivíduos não diabéticos ${ }^{24}$. Entretanto, quando são comparados aos de outros grupos de pacientes diabéticos não portadores de feridas nos MMII, nossos valores estão abaixo daqueles encontrados em trabalhos prévios ${ }^{19,23}$. Para o teste de 2PE, não foi identificada alteração no valor obtido pareado com limiares de pacientes não-diabéticos e para 2PD não houve diferença estatística entre as pressões normais e as registradas em nosso estudo.

Nos territórios dos ramos terminais do nervo tibial posterior, os nervos plantar medial e calcâneo, os limiares encontrados foram acima do normal para IPE e IPD. Para os exames de 2PE e 2PD, o nervo plantar medial não mostrou diferença estatística significante em relação à normalidade. A sensibilidade no território do nervo calcâneo foi considerada normal para 2PE e não apresentou valores estatisticamente diferentes em relação aos valores padrão.

As medidas das distâncias observadas para a discriminação de dois pontos estáticos e dinâmicos em relação aos não-diabéticos e diabéticos sem feridas estavam acima da normalidade. Distâncias elevadas para a discriminação de dois pontos refletem significativa quantidade de fibras nervosas degeneradas, uma vez que esta modalidade de exame avalia a densidade de fibras nervosas ${ }^{34,39}$. As medidas na região da polpa do hálux e pele medial do calcâneo também sugerem ter havido 


\section{Figura 2 - Programa de acompanhamento da neuropatia diabética pelo PSSD $^{\text {TM }}$ em pacientes não-portadores de feridas nos MMII}

NEUROPATIA DIABÉTICA

(NÃO PORTADOR DE FERIDA MMII)

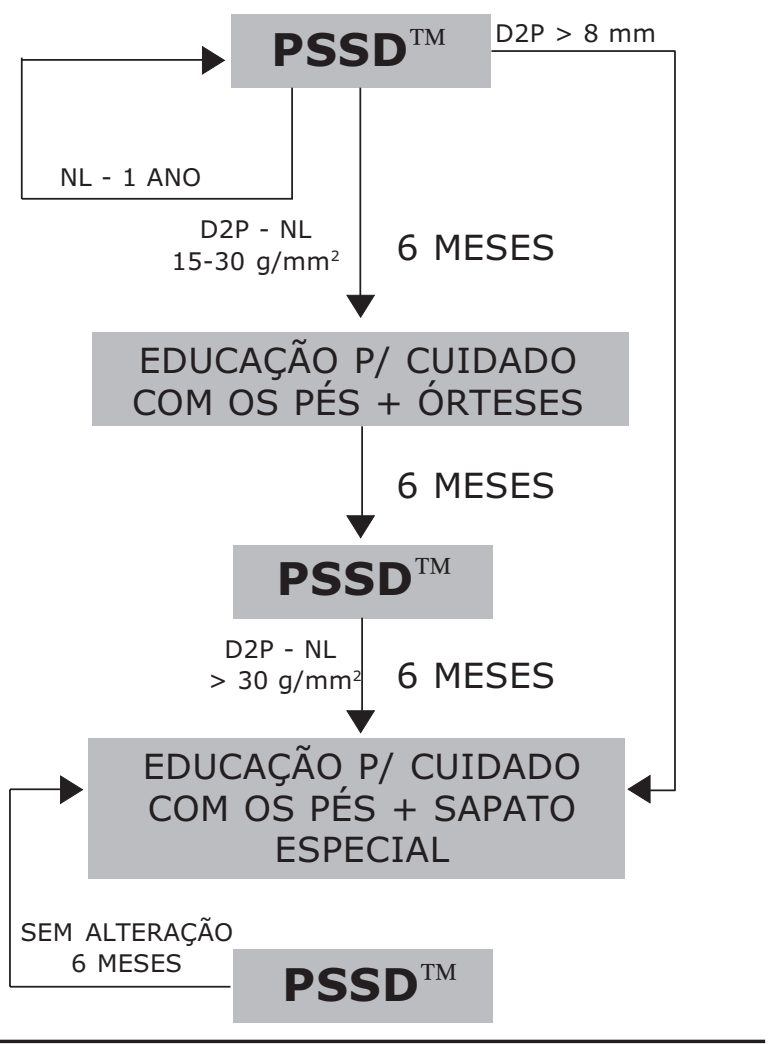

importante perda de axônios, uma vez que a discriminação de dois pontos só foi sentida a partir de $1 / \mathrm{mm}$.

Ao compararmos os valores obtidos do nosso estudo às estimativas de pressão fornecidas pelo monofilamento de Semmes-Weinstein ${ }^{\circledR}$ (Tabela I), demonstramos que a utilização de dispositivo capaz de quantificar o limiar mínimo de pressão cutânea nos permite, não somente, avaliar a função do conjunto fibras de adaptação lenta e rápida e seus respectivos receptores, como nos concede a oportunidade de detectar estágios precoces da evolução da neuropatia diabética acompanhando o déficit funcional da fibra nervosa.

A fim de identificar inicialmente e acompanhar, de modo mais rigoroso, a evolução da neuropatia diabética, sugerimos seguimento prospectivo por meio do teste realizado com o PSSD ${ }^{\mathrm{TM}}$, determinado na Figura 2.

\section{CONCLUSÃo}

Complicações decorrentes do Diabetes mellitus nos MMII têm aumentado significantemente custos médico-hospitalares tanto do sistema privado como público de saúde em todo o mundo.
Especialistas recomendam identificação precoce da perda de sensibilidade protetora, a fim de se evitar as consequências dramáticas impostas pela ulceração ou até mesmo amputação destes membros.

O PSSD ${ }^{\text {TM }}$ oferece o principal critério para avaliação da neuropatia; é capaz de quantificar e expressar o limiar mínimo de sensibilidade cutânea. Possibilita evolução mais precisa e contínua da função do conjunto fibra/receptor de adaptação lenta e rápida em um só equipamento, sem necessidade de vários dispositivos que apenas fornecem resposta dicotômica.

\section{Conflito de interesse: não há}

\section{SUMMARY}

Cutaneous sensibility threshold in the feet of Diabetic PATIENTS WITH PRESSURE SPECIFIED SENSORY DEVICE: AN ASSESSMENT OF THE NEUROPATHY

OBJECTIVES. Neuropathy is a severe progressive loss of protective sensation in the feet, increasing patient vulnerability to mechanical trauma and consequently more prone to development of chronic wounds, major distortion of the foot bone architecture and to eventual limb amputation. Prophylaxis should be enforced to avoid foot ulceration and for this purpose, evaluation of the degree of loss of sensation on the skin is essential. The PSSD (Pressure Specified Sensory Device ${ }^{T M}$ ) was developed to quantify the threshold of pressure applied to the skin that the patient might recognize as positive. Pressure of one or two points is tested both statically and with movement, thus assessing the function of fast and slow response nerve fibers.

METHODS. 33 diabetic patients, type II, with no previous history of wounds on the lower extremity were studied. The tests used were, one point static, one point moving and two points, static and moving on the cutaneous territory of the fibular nerve and posterior tibial nerve (two territories - medial plantar and calcaneous nerves).

RESULTS. Altered values were observed for the static and dynamic tests over the three nerve territories studied. Differences were statically significant $(p<0.05)$. This numeric quantification of the threshold of pressure supports the evaluation of the status of the fiber/receptor structures as well as the functional deficit subsequent to diabetic neuropathy. [Rev Assoc Med Bras 2009; 55(I): 29-34]

KEY wORDS: Diabetic neuropathy. Cutaneous sensibility. Assessment method.

\section{REFERÊNCIAS}

I. Pham H, Armstrong DG, Harvey C. Screening techniques to identify people at high risk for diabetic foot ulceration. Diabetes Care, 2000;23:606-II .

2. Dyck PJ, Thomas PK, Lambert EH, Bunge R. Peripheral neuropathy, 2nd ed. Philadelphia: W.B. Saunders Company; 1984.

3. Dellon AL. Treatment of symptoms of diabetic neuropathy by peripheral nerve descompression. Plast Reconstr Surg. 1992;89:689-97.

4. Siitonen OI, Niskanen LK, Laakso M. Lower extremity amputations in diabetic and nondiabetic patients. Diabetes Care. 1993; 16:16.

5. Clark CM, Lee A. Prevention and treatment of the complications of diabetes mellitus. N Engl J Med. 1995;332: I 1 10-7.

6. Holewski J. Aesthensiometry: quantification of cutaneuos pressure sensation in diabetic peripheral neuropathy. J Rehabil Res Dev. 1988;25: I - 10. 
Carvalho VF et al.

7. Sosenko JM, Kato M, Soto R. Comparison of quantitative sensory threshold measures for their association with foot ulceration in diabetic patients. Diabetes Care. 1990; 13: | 057-6I.

8. Altman MI, Altman KS. The podiatric assessment of the diabetic lower extremity: special considerations. Wounds. 2000; I (Suppl B):64B-7IB.

9. Boike AM, Hall JO. A practical guide for examining and treating the diabetic foot. Cleve Clin J Med. 2002;69:342-8.

10. Singh N, Armstrong DG, Lipsky BA. Preventing foot ulcers in patients with diabetes. JAMA. 2005;293:217-28.

I I. Dellon ES, Mourey MS, Dellon AL. Human pressure perception values of constant and moving one and two-point discrimination. Plast Reconstr Surg. 1991;90:1 12-7.

12. Omer GE Jr. Methods of assessment of injury and recovery of peripheral nerves. Surg Clin North Am. 1981;61:303-19.

13. Levin S, Pearsall G, Ruderman RJ. Von Frey's method of measuring pressure sensibility in the hand: an engineering analysis of the WeinsteinSemmes pressure aesthesiometer. J Hand Surg. 1978;3:21 I-6.

14. Malgrange D, Richard JL, Leymarie F. Screening diabetic patients at risk for foot ulceration. A multi-centre hospital-based study in France. Diabetes Metab. 2003;29:261-8.

15. Dellon AL. Clinical use of vibratiry stimuli to evaluate peripheral nerve injury and compression neuropathy. Plast Reconstr Surg 1980;65:466-76.

16. Armstrong DG, Hussain SK, Middleton J, Peters EJ, Wunderlich RP, Lavery LA. Vibration perception threshold: are multiple sites of testing on diabetes foot examination? OstomyMound Management. 1998;44:70-6.

17. Dellon ES, Mourey R, Dellon AL. Human pressure perception values for constant and moving one and two point discrimination. Plast Reconstr Surg. 1992;90: I 12-7

18. Ferreira MC, Costa MP, Cunha MS, Sakae E, Fels KW. Sensibility of the brest after reduction mammaplasty. Ann Plast Surg. 2003;5 I: I-5.

19. Ferreira MC, Rodrigues L, Fels KW. New mwthod for evaluation of cutaneous sensibility in diabetic feet. Preliminary report. Rev Hosp Clin Fac São Paulo.2004;59: I - 10

20. Fels KW, Cunha MS, Sturtz GP, Gemperli R, Ferreira MC. Evaluation of cutaneous abdominal wall sensibility after abdominoplasty. Aesthetic Plast Surg. 2005;29:78-82.

21. Fogaça WC, Sturtz GP, Surjan RC, Ferreira MC. Evaluation of cutaneous sensibility on infraorbital nerve area. J Craniofac Surg. 2005; 1 6:953-6.

22. Szklo M, Nieto FJ. Epidemiology: beyound the basics. Gaithersburg:Aspen; 2000. p.33-8.

23. Sociedade Brasileira de Diabetes. Tratamento e acompanhamento do diabetes mellitus: diretrizes da Sociedade Brasileira de Diabetes. [citado 2008]. Rio de Janeiro: Diagraf; 2006. Disponível em: http:// www.diabetes.org.br/educacao/docs/diretrizes.pdf.

24. Tassler PL, Dellon AL, Scheffler NM. Cutaneous pressure threshold in diabetics with and without foot ulceration: evaluation of sensibility with the Pressure Specified Sensory Device. I Am Podiatric Med Assoc. 1995;85:679-84
25. Forouzandeh F, Ahari AA, Abolhasani F, Larijani B. Comparison of different screening tests for detecting diabetic foot neurophaty. Acta Neurol Scand. 2005; I 12:409-13.

26. American Diabetes Asssociation. Consensus development conference on diabetic foot wound care. Diabetes Care. 1999;22:1354-60.

27. Larijani B, Forouzandeh F. Diabetic foot disorders, a review article. Iran J Diabetes Lipid Disord. 2003;2:93-I 03.

28. Consensus Statment. Report and recommendations of the San Antonio Conference on diabetic neuropathy. Diabetes Care 1988; | 1:592-7.

29. Armstrong DG. The 10-g monofilament: the diagnostic divining rod for the diabetic foot? J Foot Ankle Surg. 1999:69:342-8.

30. Barber MA, Conolley J, Spaulding CM, Dellon AL. Evaluation of pressure threshold prior to foot ulceration. I Am Podiatr Med Assoc. 2001;91:508-14.

31. McGill M, Molyneaux L, Spencer R. Possible sources discrepancies in the use of the Semmes-Weinstein monofilament: impact on prevalence of insensate foot and workload requirements. Diabetes Care. 1999;22:598-602.

32. Armstrong DG, Lavery LA, Vela AS. Choosing a practical screening instrument to identify patients at risk for diabetic foot ulceration. Arch Intern Med. 1998; 158:289-92.

33. Smieja M, Hunt DL, Edelman D. International Cooperative Group for Clinical Examination Research. Clinical examination for the detection of protective sensation in the feet of diabetic patients. J Gen Intern Med. 1999; | 4:4 18-24.

34. Dellon AL, Mckinnon SE, Brandt KE. The markings of the SemmesWeinstein nylon monofilaments. J Hand Surg. 1993; 18:756-7.

35. Levin S, Pearsall G, Ruderman RS. J Hand Surg. 1978:3 apud Dellon AL. von Frey's Method of measuring pressure sensibility in the hand: an engineering analysis of the Weinsten-Semmes pressure aesthesiometer. In: Somatosensory testing and rehabilitation. Baltimore 2000. p.99-I75.

36. Weber EH. Veber den Tastinn. Arch Anat Physiol Wissensch Méd 1835; 152 apud Wilgis EFS. Techiniques for diagnosis of peripheral nerve loss. Clin Orthop. 1982; 163:1 I.

37. Moberg E. Objetive methods for determining the functional value of sensibility in the hand. J Bone Joint Surg 1958;40:454 apud Wilgis EFS. Techiniques for diagnosis of peripheral nerve loss. Clin Orthop. 1982; 163:11.

38. Mackinnon SE, Dellon LD. Two-point discrimination tester. J Hand Surg. 1985; 10A:906-7.

39. Wood WA, Wood MA, Weter SA, Menn JJ, Hamilton SA, Dellon AL. Testing for loss of protective sensation in patients with foot ulceration. J Am Podiatr Med Assoc. 2005;95:469-74.

Artigo recebido: 07/03/07

Aceito para publicação: 10/05/08 